Tropical Journal of Pharmaceutical Research September 2019; 18 (9): 1811-1815

ISSN: $1596-5996$ (print); 1596-9827 (electronic)

(c) Pharmacotherapy Group, Faculty of Pharmacy, University of Benin, Benin City, 300001 Nigeria.

\title{
Quercetin attenuates lipopolysaccharide-induced myocardial cell apoptosis via modulation of cAMP-Epac pathway
}

\author{
YuHui Wang ${ }^{1 *}$, Qian $\mathrm{Fu}^{2}$, Baning Ye ${ }^{1}$, Yanpei Liü \\ ${ }^{1}$ Intensive Care Unit, Guizhou Provincial People's Hospital, Guiyang City, ${ }^{2}$ Psychological \& Counseling Center, Guizhou \\ University, No. 2708, South Section of Huaxi Avenue, Huaxi District, Guiyang City, Guizhou Province 550002, China
}

*For correspondence: Email: wangyuhuijhdld@163.com; Tel: 0086-851-85625209

\begin{abstract}
Purpose: To investigate the effects and mechanism of action of quercetin (QUE) on sepsis-induced apoptosis of myocardial cells in vitro.

Methods: Lipopolysaccharide (LPS) was used to induce apoptosis H9c2 myocardial cells. Apoptosis of H9c2 cells was determined by propidium iodide staining. Knock down of Epac1 was achieved using small interfering RNA (SiEpac1). The levels of associated proteins (Epac1 and Rap1) were evaluated by western blotting.

Results: Lipopolysaccharide promoted apoptosis of H9c2 cells and inhibited the activity of CAMP-Epac pathway ( $p<0.001$ vs. control). Quercetin inhibited caspase 3 activity and apoptosis $(p<0.05$ vs. LPS) induced by LPS via activation of CAMP-Epac1 signaling pathway. Moreover, Epac1 knockdown decreased the anti-apoptosis effect of Que, which indicates that Que attenuated apoptosis partly via cAMP-Epac pathway.

Conclusion: Que attenuated LPS-induced apoptosis in myocardial cells via activation of CAMP-Epac1 pathway. Therefore, quercetin treatment may serve as a promising strategy in the treatment of sepsisinduced myocardial injury.
\end{abstract}

Keywords: Quercetin, cAMP-Epac pathway, Apoptosis, Sepsis, Myocardial injury

\begin{abstract}
This is an Open Access article that uses a fund-ing model which does not charge readers or their institutions for access and distributed under the terms of the Creative Commons Attribution License (http://creativecommons.org/licenses/by/4.0) and the Budapest Open Access Initiative (http://www.budapestopenaccessinitiative.org/read), which permit unrestricted use, distribution, and reproduction in any medium, provided the original work is properly credited.

Tropical Journal of Pharmaceutical Research is indexed by Science Citation Index (SciSearch), Scopus, International Pharmaceutical Abstract, Chemical Abstracts, Embase, Index Copernicus, EBSCO, African Index Medicus, JournalSeek, Journal Citation Reports/Science Edition, Directory of Open Access Journals (DOAJ), African Journal Online, Bioline International, Open-J-Gate and Pharmacy Abstracts
\end{abstract}

\section{INTRODUCTION}

Sepsis, a leading cause of death worldwide, is a systemic dysregulated host response caused by infection and may lead to multiple organ dysfunction syndrome (MODS) [1]. Organ dysfunction is considered an important characteristic of severe sepsis, and recent research has shown the importance of using myocardial depression and cardiac dysfunction as criterion for diagnosis of severe sepsis [2]. About $50 \%$ of patients with sepsis have myocardial injury [3]. Myocardial cell apoptosis, which is associated with sepsis-induced myocardial injury, is believed to be an important mechanism of myocardial dysfunction [4].

Quercetin (Que, 3,5,7,3',4'-pentahydroxyflavone), a potent plant-derived flavonoid, exerts various physiological effects including anti-oxidation, 
anti-inflammation, and anti-proliferation [5,6]. Studies have demonstrated the protective effects of Que on multiple organs in inflammatory disorders, such as myocardial diseases[7]. Moreover, intake of Que has been correlated with reduced incidence of cardiovascular diseases [8].

Myocardial cell death is one of the main causes of heart failure induced by sepsis. Previous research demonstrated that Que pretreatment protect myocardial cells against ischemiareperfusion injury via anti-oxidation, antiinflammation, and anti-apoptotic mechanisms in vivo through the PI3K/Akt pathway[9].Although the protective effects of Que in myocardial cells were previously reported, the mechanism by which Que protects cells from sepsis-induced myocardial cell apoptosis remains poorly understood. This study investigated the effects of Que on lipopolysaccharide (LPS)-induced apoptosis of myocardial cells, and the associated mechanism.

\section{EXPERIMENTAL}

\section{Chemicals}

Primary antibodies against caspase 3 (no. \#9662), Epac1 (no. \#4155), Rap1 (no. \#2326), and tubulin (no. \#2128) were purchased from Cell Signaling Technology, Inc. (Beverly, MA, USA). Lipopolysaccharide (no. L2880) was purchased from Sigma-Aldrich (St Louis, MO, USA). Quercetin (no.100081, purity exceed 98 $\%$ ) was purchased from National Institutes for Food and Drug Control (Beijing, China).

\section{Cell culture}

The H9c2 cells were purchased from the ATCC (Rockville, MD, USA) and cultured in DMEM supplemented with 10\% FBS, 2 mM glutamine, and $1 \mathrm{mM}$ HEPES buffer, according to manufacturer's instructions. The cells were incubated in FBS-free basal medium overnight before treatment.

\section{Apoptosis assay}

Apoptosis of $\mathrm{H} 9 \mathrm{c} 2$ cells was analyzed by propidium iodide $(\mathrm{PI})$ staining. Cells were treated with LPS plus Que or 8-pCPT-2'-O-Me-cAMP (8pCPT, Epac1 agonist) for $24 \mathrm{~h}$. After another 24 $\mathrm{h}$, the cells were collected and fixed in cold ethanol $(70 \%)$, stained with $\mathrm{Pl}$ for $30 \mathrm{~min}$ in the dark, and PI-positive cells (apoptotic cells) were identified using a fluorescence microscope (excitation at $535 \mathrm{~nm}$, and emission at $615 \mathrm{~nm}$ ).

\section{Determination of caspase-3 activity}

The activity of caspase- 3 was measured using a EnzChek Caspase-3 Assay Kit (Thermo Fisher, Scotts Valley, CA, USA). About $1 \times 10^{6}$ cells for each group were collected and lysed with lysis buffer. After centrifugation, the reaction buffer and substrate solution were added to each sample sequentially. The activity of caspase-3 was determined using a fluorescence microplate reader (excitation/emission 342/441 nm).

\section{Epac1 knockdown by siRNA transfection}

Small interfering RNA against Epac1 (siEpac1) was synthesized by Invitrogen (Carlsbad, CA, USA). H9c2 cells were transfected with siEpac1 or negative control using Lipofectamine 2000 (Invitrogen).

\section{Western blotting}

The cells of different groups were collected and lysed in RIPA buffer with protease and phosphatase inhibitor cocktails. Protein concentrations were measured by Bradford method (Thermo Scientific, Rockford, IL, USA). Proteins were separated and transferred to polyvinylidene difluoride membranes. After blocking, the membranes were incubated with the respective primary antibodies (at a dilution of $1: 1000)$, and subsequently with secondary antibodies $(1: 10,000)$. The proteins were detected using an enhanced chemiluminescence system.

\section{Statistical analysis}

Data were analyzed by one-way ANOVA using Prism 6 (GraphPad Software CA, USA), and are presented as mean \pm standard deviation (SD).

\section{RESULTS}

\section{LPS promoted apoptosis in $\mathrm{H} 9 \mathrm{c} 2$ cells and inhibited activation of cAMP-Epac}

As shown in Figure $1 \mathrm{~A}$, the number of apoptotic cells $\left(\mathrm{Pl}^{+}\right)$was increased in the LPS group (about seven-fold more than control; $p<0.001$ ), and the 8-pCPT Epac1 agonist inhibited LPS-induced apoptosis. The activity of caspase-3 (Figure $1 \mathrm{~B}$ ) was increased about 12-fold when compared with control $(p<0.001)$ but was inhibited by 8 pCPT ( $p<0.01$ vs. LPS group). The expressions of cAMP-Epac signaling proteins (Epac1 and Rap1) were decreased in the LPS group, and 8pCPT reversed the effects of LPS. 
A
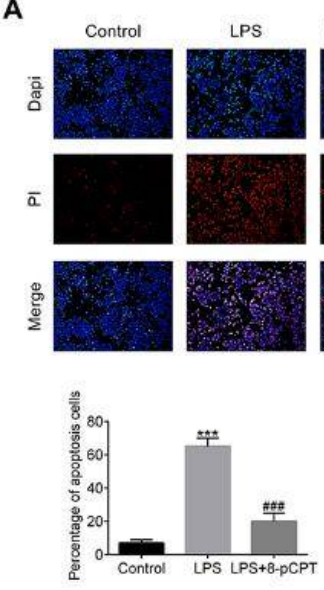

B
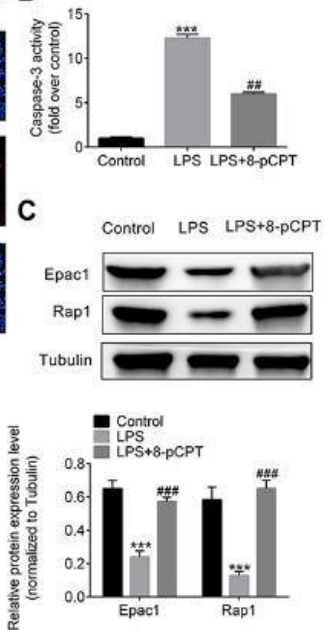

Figure 1: Apoptosis and associated protein expressions of LPS-treated H9c2 cells. (A) Apoptosis analysis by $\mathrm{PI}$ staining of $\mathrm{H} 9 \mathrm{c} 2$ cells. (B) Activity of caspase-3 in the LPS-treated $\mathrm{H} 9 \mathrm{c} 2$ group with or without 8-pCPT treatment. (C) expressions of Epac1 and Rap1 in theH9c2 group measured by western blotting; $p<0.001$ vs. control, ${ }^{\# \#} p<0.01$, ${ }^{\# \# \# ~} p<$ 0.001 vs.control group

\section{Que inhibited LPS-triggered H9c2 cell apoptosis}

To investigate the effects of Que on $\mathrm{H} 9 \mathrm{c} 2$ cell apoptosis, the cells were treated with 10,30 , or $60 \mu \mathrm{M}$ Que after LPS treatment. The results are shown in Figure 2. Propidium lodide staining indicated that Que inhibited LPS-triggered H9c2 cell apoptosis, and the number of apoptotic cells in the $30 \mu \mathrm{M}$ Que group decreased the most $(p<$ $0.01)$. The activity of caspase-3 decreased by about $50 \%$ in the Que group $(30 \mu \mathrm{M})$ when compared with the LPS group ( $p<0.01)$, which was in agreement with the apoptosis results.

\section{Que promotes activation of CAMP-Epac1 signaling pathway}

To investigate the mechanism of Que on H9c2 cell apoptosis induced by LPS, the concentration of cAMP, and expressions of Epac1 and Rap1 were evaluated and the results are shown in Figure 3. The levels of cAMP, Epac1 and Rap1 were decreased by over $50 \%(p<0.001$ for cAMP, and $p<0.01$ for Epac1 and Rap1) when compared with the control group. Quercetin treatment increased the levels of cAMP, Epac1and Rap1. Furthermore, 8-CPT markedly increased the expression of Epac1, and Rap1 in LPS-stimulated $\mathrm{H} 9 \mathrm{c} 2$ cells, which was similar to the effects of Que treatment. Together, these results demonstrated that Que promoted the activation of CAMP-Epac1 signaling.

A
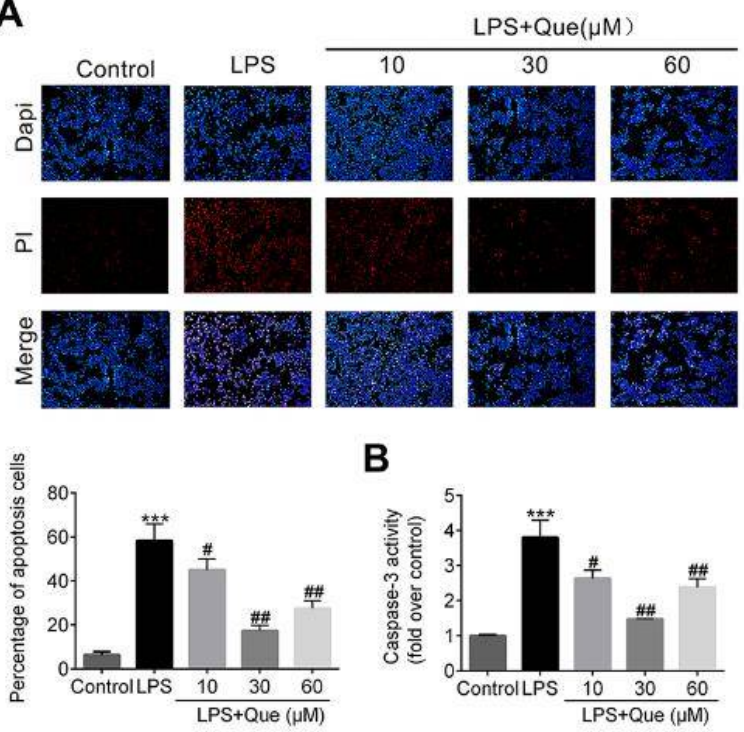

B

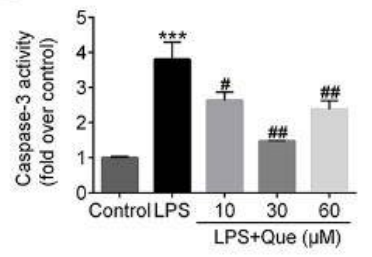

Figure 2: Effect of Que on LPS-induced H9c2 cell apoptosis. (A) Apoptosis of H9c2 cells was evaluated by Propidium lodide staining. (B) Effect of QUE on caspase-3; $" p<0.05,{ }^{* *} p<0.01$, and ${ }^{* *} p<0.001$, compared with controls
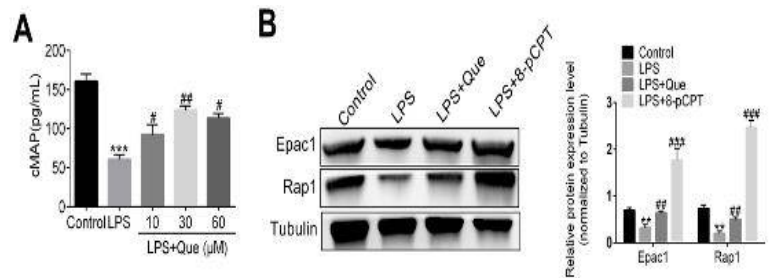

Figure 3: cAMP level $(A)$ and expression levels of Epac1 and Rap1 (B). Relative protein expressions are shown in the right panel; ${ }^{* *} p<0.01$ and ${ }^{* *} p<0.001$ vs. control, ${ }^{\# \#} p<0.01$ and ${ }^{\# \# \#} p<0.001$ vs. control group

\section{QUE inhibited LPS-induced apoptosis of H9c2 cells via Epac1 pathway}

To further investigate the role of Epac1 in the inhibitory effects of Que on apoptosis, siRNA was used to knockout the Epac1 proteins, and the cells were then treated with LPS with or without the addition of Que. As shown in Figure 4 $A$, the number of apoptotic cells was increased in the LPS group and the effects were inhibited by Que. However, the inhibitory effects of Que on apoptosis were reversed by Epac1 knockout. The activity of caspase- 3 is shown in Figure $4 \mathrm{~B}$. Quercetin treatment decreased the LPS-induced activity of caspase-3, and Epac1 knockout partly inhibited the effects of Que. The levels of Epac1 and Rap 1 are shown in Figure 4 C. Lipopolysaccharide-induced increased expressions of Epac1 and Rap 1, and Que treatment enhanced the effects of LPS. However, the effect of Que was inhibited in Epac1 knockout cells. Therefore, Que inhibited the LPS- 
induced apoptosis of H9c2 cells partly through the Epac1 pathway.

A

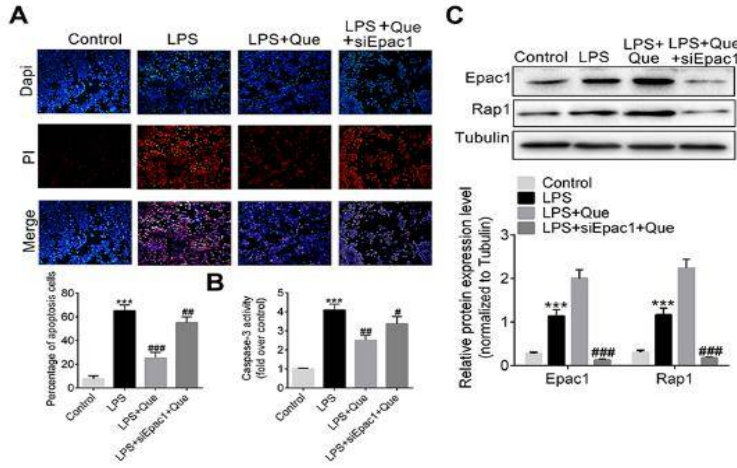

Figure 4: Apoptosis and associated protein expressions. (A) Apoptosis analysis by PI staining of $\mathrm{H} 9 \mathrm{c} 2$ cells. (B) Caspase-3 activity in H9c2 cells (transfected with siEpac1) treated with LPS with or without Que. (C). Expression of Epac1 and Rap1 in $\mathrm{H} 9 \mathrm{c} 2$ cells, as measured by western blotting. Quantitative scans are shown in the bottom right panel; ${ }^{* * t} p<0.001$ vs. control, ${ }^{\# \#} p<0.01$ and ${ }^{\# \# \#} p<$ 0.001 vs. control group

\section{DISCUSSION}

Myocardial injury is a common complication of sepsis and septic shock, with an incidence rate of up to $40 \%$. Myocardial injury is also an important cause of poor prognosis of sepsis [4]. Many factors lead to myocardial injury in sepsis, including cytokines, oxidative stress, calcium overload, and excessive activation of the renin angiotensin system (RAS) [10]. Recent studies indicated that apoptotic pathways are activated by LPS, which plays a vital role in the myocardial injury caused by sepsis [11]. In the present study, LPS was used to induce apoptosis of H9C2 cells to model sepsis-induced myocardial injury. The number of apoptotic cells and the level of pro-apoptotic protein caspase3wereincreased after LPS treatment.

As a common second messenger, cAMP, which regulates many cellular processes, is a key signaling molecule in the cardiovascular system and the myocardial injury response pathway [12].Cyclic adenosine monophosphate is involved in a variety of cellular processes mostly through activation of protein kinase $A$ (Epac) [13]. Epac isoforms, Epac1 and Epac2, are considered to be the important downstream components of the cAMP pathway due to their ability in activating small GTPase Rap [14]. Elevated intracellular cAMP has been shown to produce protective effects, both in clinical observations and animal experiments, and in various pathological situations such as during ischemia/reperfusion injury [15]. Xin Wang et al demonstrated that the Epac1-Rap1 pathway is involved in the pathogenesis of myocardial ischemia/reperfusion injury in vitro and in vivo [16].The present study is in agreement with previous reports that showed that the levels of cAMP, Epac1, and Rap1 were all decreased after LPS treatment. The CAMP analogs, including 8-pCPT, are selective activators of Epac $[13,17]$. In this study, the Que-triggered effects of apoptosis and CAMP-Epac1 signaling were similar to 8-pCPT treatment.

Moreover, the effects of Que on sepsis in vivo have been reported. Que ameliorates systemic inflammation due to LPS-induced sepsis of mice via promoting the expression of IL-10 (an antiinflammatory cytokine) [18]. Quercetin alleviated sepsis-induced cardiac insufficiency in mice and improved survival rates via regulating nitrous oxide production and the apoptosis pathway [19].Quercetin also exerts protective effects on other organ dysfunctions induced by sepsis. Chang et al found that Que protect mice from LPS-induced sepsis via the NF-kB signaling pathway, and reduced the inflammatory response of tissues [20]. In vivo experiments are needed to further confirm the effects of Que on sepsis-induced myocardial injury.

\section{CONCLUSION}

Quercetin attenuates LPS-induced apoptosis of myocardial cells via activation of cAMP-Epac1 pathway, which highlights the protective effects of Que against myocardial injury induced by sepsis.

\section{DECLARATIONS}

\section{Conflict of interest}

No conflict of interest is associated with this work.

\section{Contribution of authors}

We declare that this work was done by the authors named in this article and all liabilities pertaining to claims relating to the content of this article will be borne by the authors. YuHui Wang designed all the experiments and revised the paper. Qian $\mathrm{Fu}$ performed the experiments. While Baning $\mathrm{Ye}$ and Yanpei Liu wrote the manuscript.

\section{Open Access}

This is an Open Access article that uses a funding model which does not charge readers or their 
institutions for access and distributed under the terms of the Creative Commons Attribution License (http://creativecommons.org/licenses/by/ 4.0) and the Budapest Open Access Initiative (http://www.budapestopenaccessinitiative.org/rea d), which permit unrestricted use, distribution, and reproduction in any medium, provided the original work is properly credited.

\section{REFERENCES}

1. Jain S. Sepsis: An Update on Current Practices in Diagnosis and Management. Am J Med Sci 2018; 356(3): 277-286.

2. C S, F S, W LD. Cardiovascular dysfunction in sepsis at the dawn of emerging mediators. Biomed Pharmacother 2017; 95(153.

3. Esper AM, Martin GS. Extending international sepsis epidemiology: the impact of organ dysfunction. Crit Care 2009; 13(1): 120.

4. W MM, C W. Sepsis and the heart. Circulation 2007; 116(7): 793-802.

5. D'Andrea G. Quercetin: A flavonol with multifaceted therapeutic applications? Fitoterapia 2015; 106(256271.

6. Huang W, Wan C, Zhou S. Quercetin-A flavonoid compound from Sarcopyramis bodinieri var delicate with potential apoptotic activity in HepG2 liver cancer cells. Trop J Pharm Res 2013; 12(4): 529-533.

7. Li C, Wang $T$, Zhang $C$, Xuan J, Su C, Wang $Y$. Quercetin attenuates cardiomyocyte apoptosis via inhibition of JNK and p38 mitogen-activated protein kinase signaling pathways. Gene 2016; 577(2): 275280.

8. Wan LL, Xia J, Ye D, Liu J, Chen J, Wang G. Effects of quercetin on gene and protein expression of NOX and NOS after myocardial ischemia and reperfusion in rabbit. Cardiovasc Ther 2009; 27(1): 28-33.

9. Liu H, Guo X, Chu Y, Lu S. Heart protective effects and mechanism of quercetin preconditioning on antimyocardial ischemia reperfusion (IR) injuries in rats. Gene 2014; 545(1): 149-155.

10. Wang D, Wu Y, Wang $X$, Liu Q, Guo Y. Mechanism of myocardial injury of sepsis and current diagnosis and treatment of TCM Beijing J Tradit Chin Med 2018; 37(2): 186-191.

11. Lancel S, Petillot $P$, Favory $R$, Stebach $N$, Lahorte $C$, Danze PM, Vallet $B$, Marchetti $P$, Neviere $R$. Expression of apoptosis regulatory factors during myocardial dysfunction in endotoxemic rats. Crit Care Med 2005; 33(3): 492-496.

12. Qin $Y$, Stokman G, Yan K, Ramaiahgari S, Verbeek $F$, de Graauw M, van de Water B, Price LS. cAMP signalling protects proximal tubular epithelial cells from cisplatininduced apoptosis via activation of Epac. $\mathrm{Br} J$ Pharmacol 2012; 165(4b): 1137-1150.

13. Enserink JM, Christensen AE, de Rooij J, van Triest $M$, Schwede F, Genieser HG, Doskeland SO, Blank JL, Bos JL. A novel Epac-specific cAMP analogue demonstrates independent regulation of Rap1 and ERK. Nat Cell Biol 2002; 4(11): 901-906.

14. Bos JL. Epac: a new cAMP target and new avenues in cAMP research. Nat Rev Mol Cell Biol 2003; 4(9): 733738.

15. Khaliulin I, Bond $M$, Suleiman M. EPAC IS A KEY MEDIATOR OF CAMP-INDUCED CARDIOPROTECTIVE SIGNALLING. Heart 2014; 100(Suppl 4): A21-A21.

16. Wang $X$, Che $X$, Jiang $Q$, Zhang $G L$, Dong $L Y$. Epac1/Rap1 signaling pathway is involved in the pathogenesis of myocardial ischemia/reperfusion injury in rats. Chinese J Pharmacol Tox 2018; v.32(04): 77-78.

17. G HG, G CO, F. S. Epac-selective cAMP analogs: New tools with which to evaluate the signal transduction properties of cAMP-regulated guanine nucleotide exchange factors. Cell Signal 2008; 20(1): 10-20.

18. Liao YR, Lin JY. Quercetin intraperitoneal administration ameliorates lipopolysaccharide-induced systemic inflammation in mice. Life Sci 2015; 137/89-97.

19. Li J, Zhang J, Dong $X$. Quercetin protects against lipopolysaccharide-induced cardiac injury in mice. $J$ South Med Univ 2015; 35(7): 1068-1072.

20. Chang YC, Tsai MH, Sheu WH, Hsieh SC, Chiang AN. The therapeutic potential and mechanisms of action of quercetin in relation to lipopolysaccharide-induced sepsis in vitro and in vivo. PLoS One 2013; 8(11): e80744. 